\title{
Thermal conductivity of crystalline AIN and the influence of atomic-scale defects
}

Cite as: J. Appl. Phys. 126, 185105 (2019); https://doi.org/10.1063/1.5097172

Submitted: 25 March 2019 . Accepted: 01 August 2019. Published Online: 12 November 2019

Runjie Lily Xu, Miguel Muñoz Rojo (D, S. M. Islam, Aditya Sood (D), Bozo Vareskic, Ankita Katre, Natalio Mingo, Kenneth E. Goodson, Huili Grace Xing (D), Debdeep Jena, and Eric Pop (D)
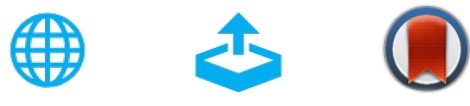

View Online

Export Citation

\section{ARTICLES YOU MAY BE INTERESTED IN}

Impact of dislocations on the thermal conductivity of gallium nitride studied by time-domain thermoreflectance

Journal of Applied Physics 126, 185103 (2019); https://doi.org/10.1063/1.5126970

Evidence of trap-assisted Auger recombination in low radiative efficiency MBE-grown IIInitride LEDs

Journal of Applied Physics 126, 184502 (2019); https://doi.org/10.1063/1.5096773

Ohmic contact to AIN:Si using graded AlGaN contact layer

Applied Physics Letters 115, 192104 (2019); https://doi.org/10.1063/1.5124936
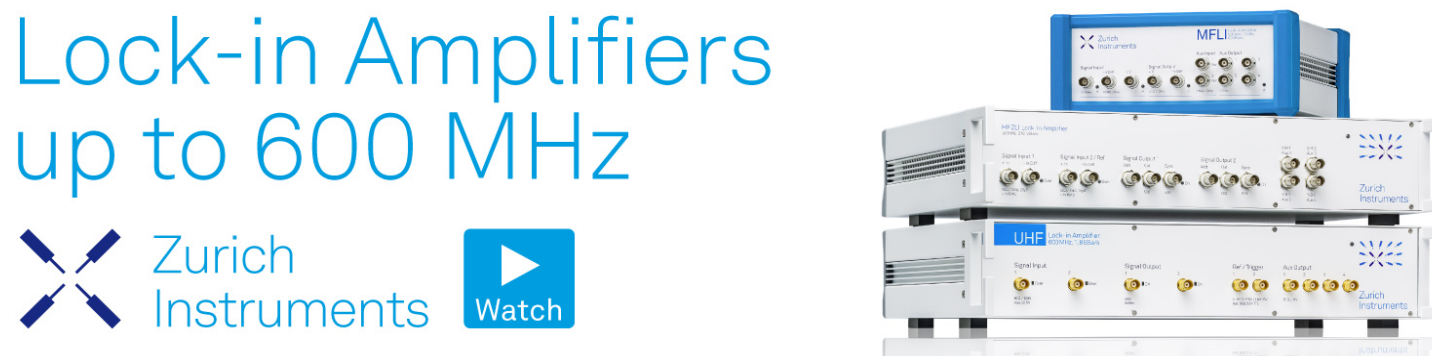


\title{
Thermal conductivity of crystalline AIN and the influence of atomic-scale defects
}

\author{
Cite as: J. Appl. Phys. 126, 185105 (2019); doi: 10.1063/1.5097172 \\ Submitted: 25 March 2019 - Accepted: 1 August 2019 . \\ Published Online: 12 November 2019
}

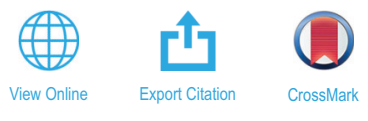

Runjie Lily Xu, ${ }^{7}$ Miguel Muñoz Rojo, ${ }^{1,2}$ (D) S. M. Islam, ${ }^{3}$ Aditya Sood, ${ }^{1,4,5}$ (D) Bozo Vareskic, ${ }^{6}$ Ankita Katre, ${ }^{7,8}$ Natalio Mingo, ${ }^{7}$ Kenneth E. Goodson, ${ }^{4,9}$ Huili Grace Xing, ${ }^{3,10}$ (iD Debdeep Jena, ${ }^{3,10}$ and Eric Pop ${ }^{1,9, a)}$ (iD)

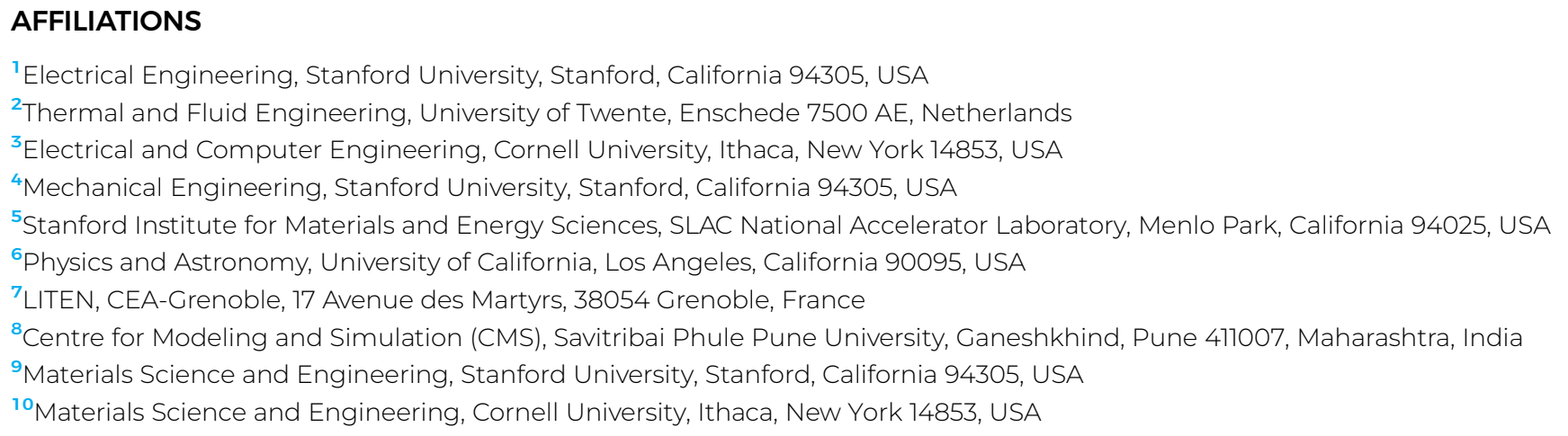

a)Email: epop@stanford.edu

\begin{abstract}
Aluminum nitride (AlN) plays a key role in modern power electronics and deep-ultraviolet photonics, where an understanding of its thermal properties is essential. Here, we measure the thermal conductivity of crystalline AlN by the $3 \omega$ method, finding that it ranges from $674 \pm 56 \mathrm{Wm}^{-1} \mathrm{~K}^{-1}$ at $100 \mathrm{~K}$ to $186 \pm 7 \mathrm{Wm}^{-1} \mathrm{~K}^{-1}$ at $400 \mathrm{~K}$, with a value of $237 \pm 6 \mathrm{Wm}^{-1} \mathrm{~K}^{-1}$ at room temperature. We compare these data with analytical models and first-principles calculations, taking into account atomic-scale defects ( $\mathrm{O}, \mathrm{Si}, \mathrm{C}$ impurities, and $\mathrm{Al}$ vacancies). We find that $\mathrm{Al}$ vacancies play the greatest role in reducing thermal conductivity because of the largest mass-difference scattering. Modeling also reveals that $10 \%$ of heat conduction is contributed by phonons with long mean free paths (MFPs), over $\sim 7 \mu \mathrm{m}$ at room temperature, and $50 \%$ by phonons with MFPs over $\sim 0.3 \mu \mathrm{m}$. Consequently, the effective thermal conductivity of AlN is strongly reduced in submicrometer thin films or devices due to phonon-boundary scattering.
\end{abstract}

Published under license by AIP Publishing. https://doi.org/10.1063/1.5097172

\section{INTRODUCTION}

Wide bandgap (WBG) semiconductors such as $\mathrm{GaN}, \mathrm{Ga}_{2} \mathrm{O}_{3}$, and AlN have attracted much interest due to their potential applications in power and radio frequency (RF) electronics ${ }^{1-3}$ as well as deep-ultraviolet (UV) photonics. ${ }^{4,5}$ In these contexts, heat dissipation is important during high-power and high-temperature operation. ${ }^{6-8}$ For example, power devices handle hundreds or even thousands of Volts, and the high-power density leads to high operating temperature due to Joule heating, potentially diminishing the device performance and lifetime. Thermal cycling also causes fatigue and eventual failure in such devices. ${ }^{9,10}$
Among WBG materials, AlN has a large direct bandgap $(\sim 6.1 \mathrm{eV} \text {, almost twice that of } \mathrm{SiC} \text { and } \mathrm{GaN})^{11-13}$ and one of the largest thermal conductivities. In this respect, as shown in Fig. 1, AlN is among a rare class of materials that have both a large electronic bandgap and a large thermal conductivity. AlN is widely used as a buffer for GaN growth or as a capping layer ${ }^{14,15}$ in power high-electron mobility transistors (HEMTs). However, many questions remain about the role of intrinsic defects and impurities, which can occur during AlN growth. The contribution of individual phonon modes to thermal transport in AlN is also not well understood, which is important in establishing the dependence of AlN thermal conductivity on the film thickness. 


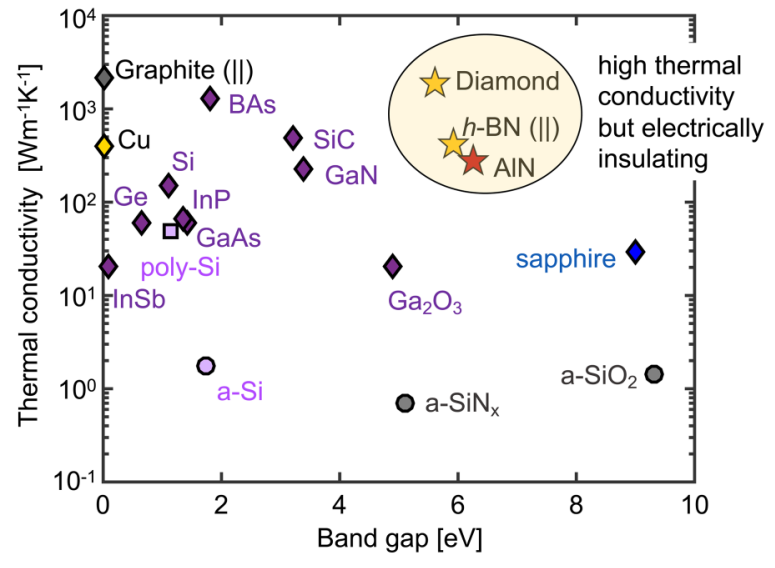

FIG. 1. Room temperature thermal conductivities of different materials vs their electronic bandgaps. These include electrical conductors (e.g., graphite and $\mathrm{Cu}$ ), ${ }^{16}$ semiconductors (e.g., $\mathrm{Si}^{16,22,23} \mathrm{Ge}^{16}{ }^{16} \mathrm{InSb},{ }^{17} \mathrm{InP},{ }^{64} \mathrm{GaAs},{ }^{17} \mathrm{BAs},{ }^{20,21}$ $\mathrm{SiC},{ }^{19} \mathrm{GaN}^{65,66}$ and $\mathrm{Ga}_{2} \mathrm{O}_{3}{ }^{67}$ ), and some electrical insulators (e.g., diamond, ${ }^{16}$ $h$-BN, ${ }^{27} \mathrm{AIN},{ }^{28,29}$ sapphire, ${ }^{24}$ amorphous $\mathrm{SiO}_{2},{ }^{25}$ and amorphous $\left.\mathrm{SiN}_{\mathrm{x}}{ }^{26}\right)$. The plot reveals that AIN lies in the same range as diamond and $h$-BN (star symbols), with both wide bandgaps and high thermal conductivities. Isotopically purified samples may have higher thermal conductivity (values displayed are for natural isotopes). Diamonds are for crystalline, squares for polycrystalline, and circles for amorphous materials.

(The contribution of electrons to thermal transport is negligible in WBG materials.)

Here, we elucidate these features of AlN thermal transport, by combining $3 \omega$ thermal measurements from 100 to $400 \mathrm{~K}$, with thermal modeling using both analytical and $a b$ initio techniques. We uncover that $\mathrm{Al}$ vacancies play an important role in limiting the thermal conductivity of present samples and that phonons with long mean free paths (MFPs $>0.3 \mu \mathrm{m}$ ) contribute over $50 \%$ of the thermal conductivity at room temperature. This implies that the effective, crystalline AlN thermal conductivity is strongly reduced in submicrometer films and could be as low as $\sim 25 \mathrm{Wm}^{-1} \mathrm{~K}^{-1}$ in a $10 \mathrm{~nm}$ thin film.

\section{MOTIVATION AND COMPARISON}

Figure 1 summarizes the room temperature thermal conductivities of several representative bulk solids with respect to their electronic bandgaps. In this plot, a few trends emerge: First, among conducting, zero bandgap materials, $\mathrm{Cu}$ and graphite (parallel to the basal plane) have the highest thermal conductivity. ${ }^{16}(\mathrm{Cu}$ is the only material on this plot whose thermal conductivity is dominated by electrons.) Second, among crystalline semiconductors, the thermal conductivity weakly scales with the electronic bandgap, ${ }^{17-19}$ as both depend on the strength of the interatomic bonds and (inversely) on the atomic mass. Crystalline boron arsenide (BAs) is somewhat of an exception, with high thermal conductivity despite a relatively moderate electronic bandgap, due to its unusual optical-acoustic phononic gap. ${ }^{20,21}$ However, polycrystalline and amorphous semiconductors (e.g., poly-Si and a-Si) have much reduced thermal conductivity due to grain boundary and disorder scattering, respectively. ${ }^{22,23}$ Third, many electrical insulators, like sapphire, $\mathrm{SiO}_{2}$ or $\mathrm{SiN}_{\mathrm{x}}$, have low thermal conductivity. ${ }^{24-26}$ Thus, only few materials have both large thermal conductivity and large electronic gap, i.e., diamond, ${ }^{16}$ hexagonal boron nitride $(h-\mathrm{BN})^{27}$ (parallel to the basal plane), and AlN, as circled in Fig. 1.

These three materials can provide excellent heat dissipation, especially in power electronics where large amounts of heat are generated. These materials can also be doped, to be used within or as parts of active device regions. The fundamental properties that lead to their high thermal conductivity are small atomic mass, strong interatomic bonds, and simple crystal structure. However, the thermal properties of AlN have been studied relatively less ${ }^{28,29}$ compared to other WBG materials, and details regarding the role of defects and phonon MFPs, particularly as a function of temperature and sample thickness, are still missing and thus the subject of this work.

\section{MEASUREMENTS AND MODELING}

\section{A. $3 \omega$ experimental measurements}

The AlN bulk crystals ( $500 \mu \mathrm{m}$ thick) were grown using physical vapor transport (PVT). ${ }^{30}$ These samples have some imperfections, including $\mathrm{Al}$ vacancies and substitutional point defects $^{31}$ of oxygen $(\mathrm{O})$, carbon $(\mathrm{C})$, and silicon $(\mathrm{Si})$ atoms, all in the range of $0.4 \times 10^{19}$ to $2 \times 10^{19} \mathrm{~cm}^{-3}$. Figure 2 shows a schematic of the $3 \omega$ setup, which is a method for thermal conductivity measurements using AC-heated electrical lines that also serve as thermometers, well described elsewhere. ${ }^{25,32,33}$ Here, four-probe metal lines ( $5 \mathrm{~nm} \mathrm{Ti}$ followed by $60 \mathrm{~nm} \mathrm{Pd}$ ) are patterned by optical lithography and lift-off on the AlN sample surface (additional information in Sec. A of the supplementary material), serving as both heaters and thermometers, as shown in Fig. 2(a). The electrical schematic of the $3 \omega$ measurement is displayed in Fig. 2(b).

As shown in Fig. 3(a), an AC current $\left(I_{1 \omega}\right)$ at frequency $\omega$ is passed through the heater, which causes a second harmonic temperature rise $\left(\Delta T_{2 \omega}\right)$ in the sample due to Joule heating. The metal heater line resistance scales linearly with temperature from $100 \mathrm{~K}$ to $400 \mathrm{~K}$, as $R=R_{0}\left[1+\alpha\left(T-T_{0}\right)\right]$, where $\alpha=(5.5 \pm 0.2) \times 10^{-3} \mathrm{~K}^{-1}$ is the temperature coefficient of resistance (TCR) and $T_{0}=100 \mathrm{~K}$, as in Fig. 3(b). Due to this linear relationship, the measured line resistance will also have a component $\left(R_{2 \omega}\right)$ that is a second harmonic of the frequency. According to Ohm's law, the heater output voltage has both $1 \omega$ and $3 \omega$ components, $V_{1 \omega+3 \omega}=R_{2 \omega} I_{1 \omega}=V_{1 \omega}+V_{3 \omega}$. We use a custom-built circuit board, schematically displayed in Fig. 2(b), to separate $V_{3 \omega}$ from $V_{1 \omega+3 \omega} \cdot{ }^{34}$ A potentiometer $\left(R_{\text {variable }}\right)$, which has a low TCR of $50 \mathrm{ppm} / \mathrm{K}$, is adjusted to match the resistance of the sample heater $\left(R_{\text {sample }}\right)$. When these two resistance values are matched, the voltage drop across the potentiometer is $V_{1 \omega}$. Both $V_{1 \omega}$ and $V_{1 \omega+3 \omega}$ are input to a lock-in amplifier, as shown in Fig. 2(b), and $V_{3 \omega}$ of the sample is the difference of these two voltage signals.

After collecting the $3 \omega$ voltage data, we analytically extract the thermal conductivity of the AlN sample as follows. The $3 \omega$ voltage $V_{3 \omega}$ vs frequency $f=\omega /(2 \pi)$ is shown in Fig. 3(c). The real part of $V_{3 \omega}$ is plotted vs $\ln (f)$ in Fig. 3(d), displaying a linear 


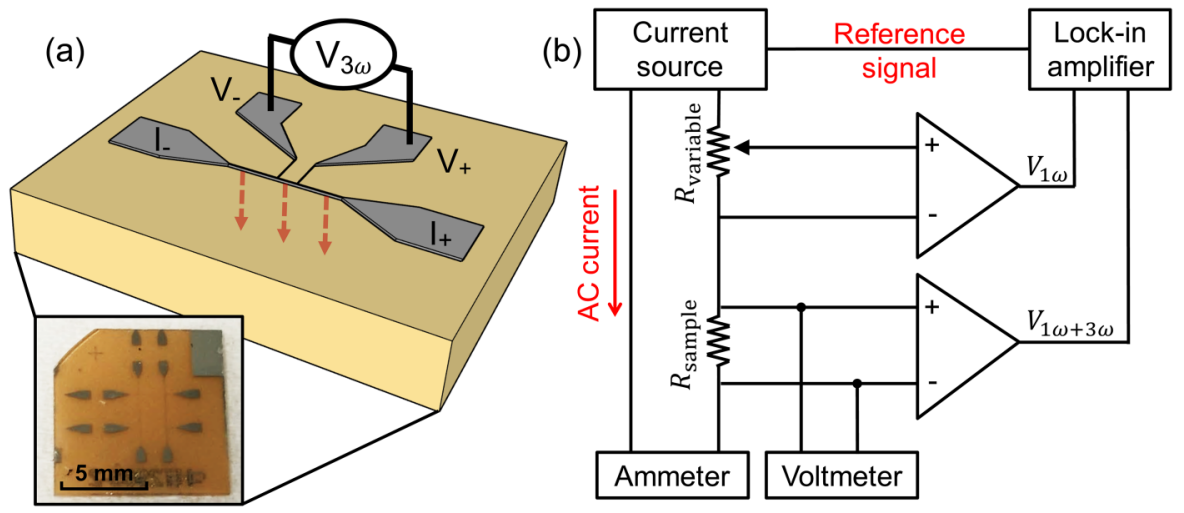

FIG. 2. (a) Schematic of four-probe $3 \omega$ metal heater line on AIN single crystal sample. Heater consists of $5 \mathrm{~nm}$ Ti and $60 \mathrm{~nm} \mathrm{Pd}, 20 \mu \mathrm{m}$ wide and $2 \mathrm{~mm}$ long between the inner voltage probes. Arrows indicate heat flow direction. Inset shows an optical image of one of the AIN samples with patterned $3 \omega$ heaters. (b) Electronic circuit and instrument setup of the $3 \omega$ measurement.

variation whose slope $S$ leads to the thermal conductivity $k$ of the sample

$$
k=\frac{R \frac{d R}{d T} I_{1 \omega}^{3}}{4 \pi L S},
$$

where $L$ is the length and $R$ is the resistance of the heater, $\mathrm{d} R / \mathrm{d} T=\alpha R_{0}$, and $I_{1 \omega}$ is the magnitude of the AC current. We used heater dimensions that were $2 \mathrm{~mm}$ long (between inner voltage probes) and $20 \mu \mathrm{m}$ wide, allowing us to treat the heater as a one-dimensional line. ${ }^{32}$ Thus, heat flow is perpendicular to the top sample surface, which is in the same direction as the (few) dislocation line defects. The density of dislocation lines provided by the manufacturer ${ }^{30}$ is in the range of $10^{2}-10^{4} \mathrm{~cm}^{-2}$, which is expected to have a small impact on the thermal conductivity. ${ }^{3.5}$

The extracted temperature-dependent thermal conductivities of two single crystal AlN samples are plotted in Fig. 4(a), from $100 \mathrm{~K}$ to $400 \mathrm{~K}$. (Sample I is shown in red diamonds and sample II is shown in blue diamonds.) All measurements were performed in a vacuum probe station $\left(<10^{-4}\right.$ Torr $)$. As a cross-check, we also used time-domain thermoreflectance (TDTR) ${ }^{36-38}$ to measure the thermal conductivity of sample II at room temperature [white diamond symbol in Fig. 4(a)], confirming the accuracy of our measurements. The average thermal conductivity of these AlN samples ranges from $674 \pm 56 \mathrm{Wm}^{-1} \mathrm{~K}^{-1}$ at $100 \mathrm{~K}$ to $186 \pm 7 \mathrm{Wm}^{-1} \mathrm{~K}^{-1}$ at (a)
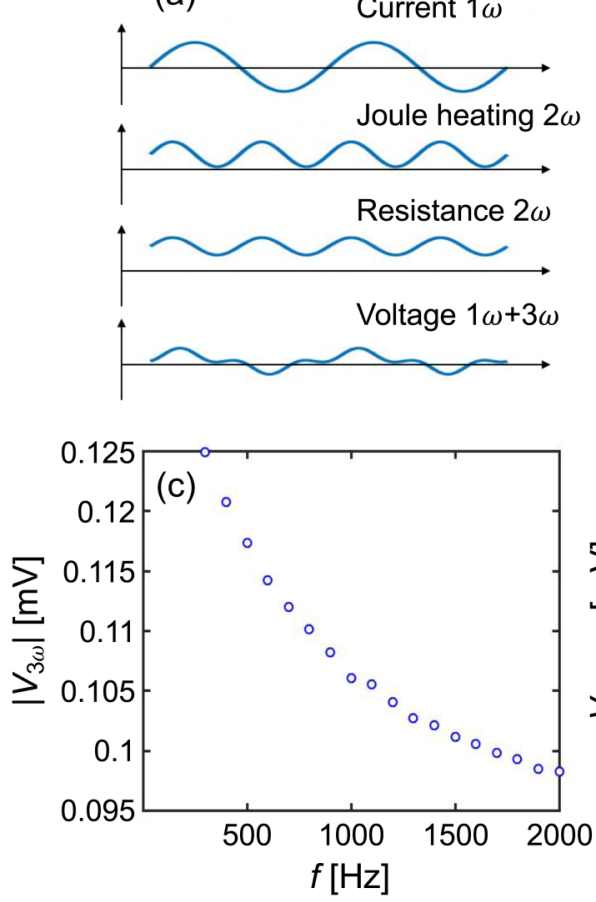
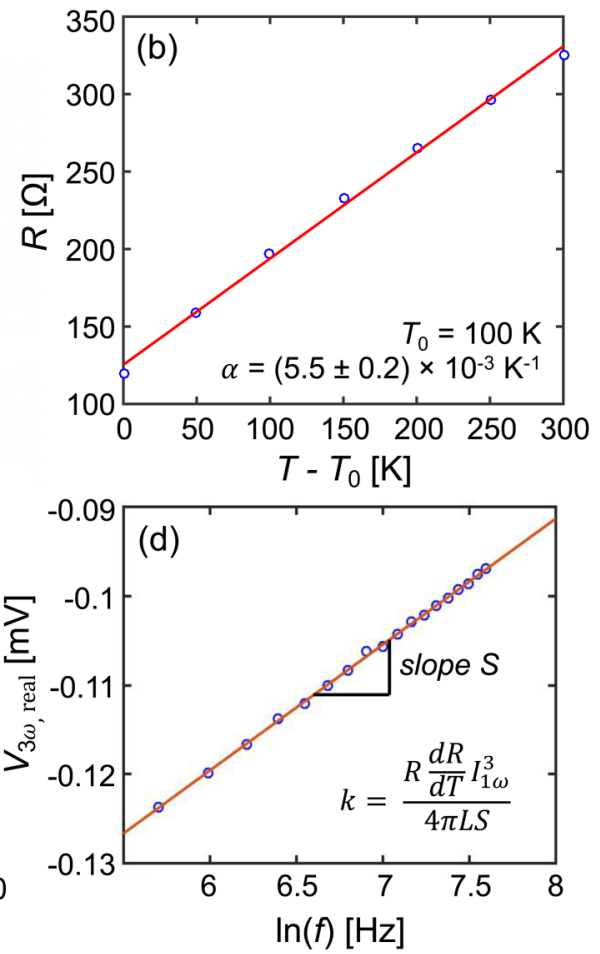

FIG. 3. Analysis of $3 \omega$ measurement. (a) An $\mathrm{AC}$ current of frequency $1 \omega$ is passed through the heater line. Joule heating causes a second harmonic temperature rise, at $2 \omega$, in the AIN sample underneath the heater. The metal heater resistance varies linearly with temperature as $R=R_{0}[1+\alpha$ $\left(T-T_{0}\right)$ ], where $\alpha$ is the TCR and $T_{0}$ is the background temperature. Due to this linear relationship, the measured heater resistance will also have a $2 \omega$ component dependent on the sample temperature. Multiplied by the $\mathrm{AC}$ current input, the output voltage will have a component at $3 \omega$. (b) TCR measurement fitting of sample I. (Sample II data are shown in Fig. S1 of the supplementary material.) Symbols are experimental data, and solid line is the fit. (c) Measured $\left|V_{3 \omega}\right|$ vs frequency $f$. The real part of $V_{3 \omega}$ is linear with $\ln (f)$, as shown in (d). Blue circles are measured data, and the thermal conductivity $k$ is calculated using the slope of the linear fit (solid line). 

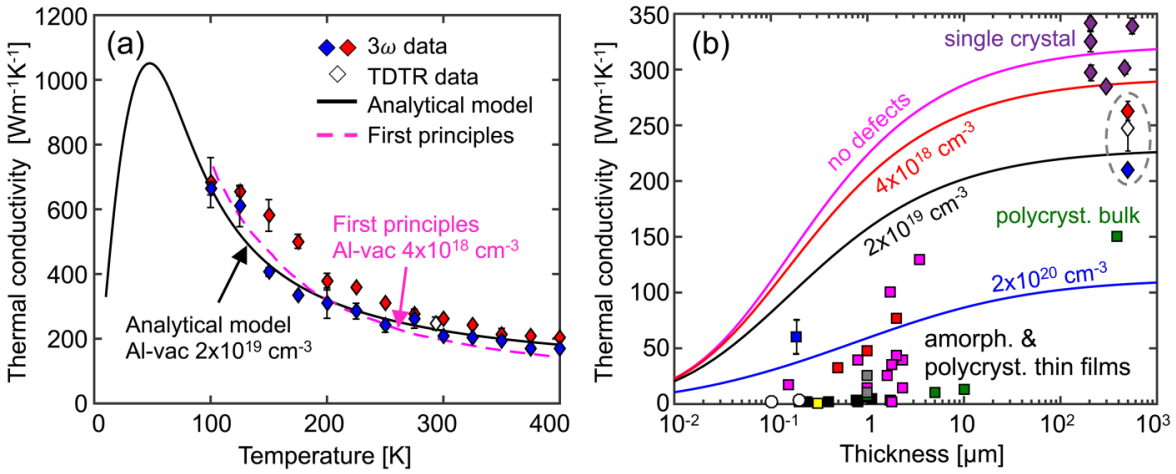

FIG. 4. (a) Thermal conductivity of AIN vs temperature. Red diamonds (sample I) and blue diamonds (sample II) are experimental data measured by our $3 \omega$ method. White diamond symbol is measured using TDTR. Dashed line is the model calculated by first-principles simulation. Solid line is the thermal conductivity calculated by the analytical model. (b) Thermal conductivity of AIN vs sample thickness at room temperature. Solid lines are the theoretical calculation using different AIN defect densities. Diamond symbols are single crystal samples measured in this work [circled, colors matching panel (a)], those by Slack et al., ${ }^{28}$ and Rounds et al. ${ }^{29}$ Square symbols are a polycrystalline bulk sample ${ }^{53}$ (in green) and various polycrystalline films (grey: Kuo et al.., ${ }^{52}$ purple: Duquenne et al., ${ }^{54}$ black: Zhao et al., ${ }^{55}$ red: Choi et al., ${ }^{56}$ blue: Yalon et al., ${ }^{5}$ yellow: Jacquot et al., ${ }^{58}$ green: Bian et al. ${ }^{59}$ ). White round symbols correspond to amorphous thin films by Zhao et al. ${ }^{55}$ and Gaskins et al. ${ }^{60}$

$400 \mathrm{~K}$. At room temperature, the average thermal conductivity is $237 \pm 6 \mathrm{Wm}^{-1} \mathrm{~K}^{-1}$ measured by the $3 \omega$ method and $247 \pm 20 \mathrm{Wm}^{-1} \mathrm{~K}^{-1}$ by TDTR (for sample II), these values being consistent with each other and similar to others reported in the literature. $^{28,29}$ We also report the thermal boundary conductance (TBC), $G_{\mathrm{b}} \approx 117 \mathrm{MW} \mathrm{m}^{-2} \mathrm{~K}^{-1}$ at room temperature between AlN and the $\mathrm{Al}$ metal pad used in TDTR, with additional details provided in Sec. B of the supplementary material. The uncertainty due to this TBC during $3 \omega$ measurements is negligible due to the large thermal diffusion length at our frequencies $(100-250 \mu \mathrm{m})$ but could play a role in thinner AlN films and devices. (The Kapitza length of AlN corresponding to this TBC is $k / G_{\mathrm{b}} \sim 2.2 \mu \mathrm{m}$ at room temperature, meaning that heat flow across AlN films thinner than this value could be partly limited by the thermal resistance of their interfaces, $1 / G_{\mathrm{b}}$.)

\section{B. Analytical model}

To analyze the contributions of different phonons and understand the underlying phonon scattering mechanisms in AlN, we turn to computational modeling, using two approaches: (1) we fit the measured data to an analytical model based on the Boltzmann transport equation (BTE) and (2) we perform full $a b$ initio calculations. The analytical model [black solid line in Fig. 4(a)] is calculated based on the simplified BTE, using the Debye approximation for the phonon dispersion of the acoustic modes (additional details are in Sec. D of the supplementary material), ${ }^{35,39}$

$$
k=\frac{1}{3} C v \lambda=\frac{1}{3} \sum_{s} \int_{0}^{\omega_{\max }} \hbar \omega g(\omega) \frac{d f(\omega, T)}{d T} v^{2} \tau(\omega) d \omega,
$$

where $\lambda$ is the phonon MFP, $v$ is the phonon group velocity, $C$ is the heat capacity, $\omega$ is the phonon frequency, $\omega_{\max }$ is the Debye cutoff frequency, $g(\omega)$ is the phonon density of states, $f(\omega, T)$ is the Bose-Einstein distribution, $\tau(\omega)$ is the phonon scattering time, and $s$ includes two transverse acoustic (TA) phonon modes and one longitudinal acoustic (LA) mode of AlN. The scattering rate is

$$
\frac{1}{\tau}=\frac{1}{\tau_{\mathrm{N}}}+\frac{1}{\tau_{\mathrm{U}}}+\frac{1}{\tau_{\mathrm{D}}}+\frac{1}{\tau_{\mathrm{B}}},
$$

where the subscripts correspond to normal-process (N), Umklapp (U), defect (D), and boundary (B) scattering, respectively. Point defect scattering arises from impurity atoms of $\mathrm{C}, \mathrm{Si}$, and $\mathrm{O}$, and from $\mathrm{Al}$ vacancies. As it turns out, the latter plays an important role in the reduction of thermal conductivity in this study, and the point defect scattering rate can be written as ${ }^{40}$

$$
\frac{1}{\tau_{D}}=\frac{V}{4 \pi v^{3}} \omega^{4} \sum_{i} f_{i}\left(\frac{m-m_{i}}{m}\right)^{2},
$$

where $V$ is the unit volume for wurtzite AlN given by $V=\sqrt{3} a^{2} c / 8$, and $a=3.11 \AA$ and $c=4.98 \AA$ are lattice constants, ${ }^{41}$ $f_{i}$ is the fractional concentration of the $i$ th impurity atom, and $m$ and $m_{i}$ are the masses of original and $i$ th impurity atoms, respectively. In point defect scattering, $\mathrm{Al}$ vacancies play a dominant role because the mass difference is the atomic mass of the $\mathrm{Al}$ atom, which is much larger than the mass difference between $\mathrm{Si}$ and $\mathrm{Al}$ atoms or the difference among $\mathrm{O}, \mathrm{C}$, and $\mathrm{N}$ atoms. In $\mathrm{AlN}, \mathrm{C}$ atoms often substitute for $\mathrm{N}$ atoms, while Si substitutes for $\mathrm{Al}^{31}$ In our analytical model, the $\mathrm{Al}$ vacancy density is used as a fitting parameter, with a fitted value of $\sim 2 \times 10^{19} \mathrm{~cm}^{-3}$, which is within the range quoted by the sample manufacturer. ${ }^{30}$ An important "shortcut" used here for treating vacancy scattering relies on a previous study by Katcho et al., ${ }^{42}$ which showed good agreement with first-principles calculations if the vacancy mass difference is taken as six times the mass of the missing atom. This is justified because vacancies lead to larger local distortion in the crystal compared to substitutional defects, due to bond breaking and atomic rearrangements, and these distortions contribute to enhanced phonon scattering. 


\section{First-principles calculations}

We also employ a second modeling approach, using first-principles calculations, based on the BTE coupled with density functional theory (DFT). This method has previously shown good agreement with experiments for a range of other materials. ${ }^{43-45}$ The phonon frequencies and anharmonic phonon scattering rates for AlN are computed using harmonic (2nd order) and anharmonic (3rd order) interatomic force constants (IFCs) for a $5 \times 5 \times 5$ supercell of AlN wurtzite structure (space group $\mathrm{P}_{3} \mathrm{mc}$ ). We follow the finite displacement method as implemented in phonopy ${ }^{46}$ and thirdorder.py, ${ }^{47}$ extracting the 2nd and 3rd order IFCs, respectively, from interatomic forces. These interatomic forces and the optimized structural parameters for wurtzite AlN are calculated using the DFT package VASP, ${ }^{48}$ and additional details are provided in Sec. E of the supplementary material. Similar to the analytic approach described earlier, the phonon scattering rate with $\mathrm{Al}$ vacancies is computed using Eq. (4), where the mass difference is six times the original atomic mass. ${ }^{42}$ All contributions to phonon scattering rates and finally the thermal conductivity are calculated using the almaBTE package, ${ }^{49}$ where the BTE is solved using an iterative scheme, and the obtained thermal conductivity is shown with a purple dashed line in Fig. 4(a), displaying good agreement with the experiments.

We note that the analytic and first-principles calculations fit the thermal conductivity data with different $\mathrm{Al}$ vacancy concentrations, i.e., $2 \times 10^{19} \mathrm{~cm}^{-3}$ and $4 \times 10^{18} \mathrm{~cm}^{-3}$, respectively, although both are in the range quoted by the sample manufacturer. ${ }^{30}$ This difference is due to the different anharmonic scattering rates implemented in the two approaches. In the analytical model, anharmonic scattering rates for both normal and Umklapp processes follow the simple $\omega^{2}$ behavior. ${ }^{28}$ The anharmonic scattering rates in the $a b$ initio calculations show deviation from this behavior at both low and high frequencies. ${ }^{44,50}$ However, we note that the five-fold difference in vacancy concentration causes only about $25 \%$ change of expected bulk thermal conductivity [Fig. 4(b)], illustrating the relative (in)sensitivity of this parameter in this range.

\section{THICKNESS DEPENDENCE OF THERMAL CONDUCTIVITY}

Figure 4(b) examines the AlN thermal conductivity dependence on vacancy concentration and film thickness. The thickness dependence with different vacancy concentrations has not been previously analyzed before, although (as we will see) AlN is subject to strong phonon-boundary scattering effects due to the large phonon MFP in this material. In other words, the thermal conductivity of submicrometer thin AlN films is strongly reduced, and thin buffer films of this material are expected to have much lower effective thermal conductivity than the bulk material. This is an "intrinsic" effect, in addition to the earlier observation of "extrinsic" thermal impedance contribution from interfaces (like Al/AlN) of submicrometer thin films.

Figure 4(b) displays the calculated thickness-dependent thermal conductivity with different defect densities using solid lines, all at room temperature. For comparison, experimental data on various single crystal films are shown in diamond symbols, including this work and Refs. 29, 51, and 52. Square symbols correspond to one bulk polycrystalline AlN measured with TDTR $^{53}$ and other polycrystalline films measured by various groups. ${ }^{52,54-59}$
Round symbols correspond to amorphous thin films by Zhao et $a l .{ }^{55}$ and Gaskins et al. ${ }^{60}$ Due to significant disorder scattering, amorphous films have much lower thermal conductivity than (poly-)crystalline films, as expected. Thus, when using AlN thin films as buffer or capping layers ${ }^{14,15}$ in power devices, highly crystalline, low-defect films provide better heat dissipation.

However, Fig. 4(b) also reveals that the thermal conductivity of all films $\sim 10 \mu \mathrm{m}$ or thinner is expected to be decreased by $\sim 10 \%$ or more from the bulk value. The effective thermal conductivities of $10 \mathrm{~nm}$ and $100 \mathrm{~nm}$ thin AlN films are predicted to be just $\sim 25 \mathrm{Wm}^{-1} \mathrm{~K}^{-1}$ and $\sim 110 \mathrm{Wm}^{-1} \mathrm{~K}^{-1}$ at room temperature (less than $1 / 12$ and $1 / 3$ of the best bulk material values), respectively, even in defect-free films, due to strong phonon-boundary scattering.

\section{ACCUMULATED THERMAL CONDUCTIVITY}

To understand the physical origin of the strong phononboundary scattering in AlN thin films, we turn to Fig. 5. First, in Fig. 5(a), we plot the calculated thermal conductivity as a function of the cumulative contributions of phonons across the range of MFPs expected in such crystals. The accumulated thermal conductivity is the thermal conductivity contribution from all phonons with MFP below a given value, ${ }^{61}$

$$
k_{\text {accum }}\left(\lambda_{0}\right)=\frac{1}{3} \sum_{s} \int_{0}^{\lambda_{0}} C(\lambda) v(\lambda) \lambda d \lambda,
$$

where $C$ is the heat capacity as a function of MFP, since $C(\omega)=\hbar \omega g(\omega) d f(\omega, T) / d T$ and $\lambda=v \tau(\omega)$. The integral is taken from 0 to $\lambda_{0}$ and thus $k_{\text {accum }}$ is the thermal conductivity of phonons with MFP $\leq \lambda_{0}$, here at room temperature. The contributions of both LA and TA modes are shown in Fig. 5(a), the LA mode contribution being larger due to its larger phonon group velocity. The total thermal conductivity is the sum of contributions from one LA and two TA modes.

To gain additional insight, we normalize the accumulated thermal conductivity by the bulk value $\left(k_{\text {accum }} / k_{\text {bulk }}\right)$ in Fig. $5(\mathrm{~b})$, for the "perfect crystal" with zero defects. Our calculations estimate that $50 \%$ of the AlN bulk thermal conductivity is contributed by phonons with MFPs $>0.3 \mu \mathrm{m}$, and $10 \%$ is contributed by phonons with very long MFPs $>7 \mu \mathrm{m}$, at room temperature. These values are comparable to the median MFP $\sim 2.5 \mu \mathrm{m}$ of Freedman et al. ${ }^{61}$ obtained by broadband frequency domain thermoreflectance (BB-FDTR), which considered only Umklapp phonon scattering (vs the four scattering mechanisms included here). Taken together, these findings explain why "size effects" on the thermal conductivity of AlN are expected to be strong in submicrometer films at room temperature, and noticeable even in sub-10 $\mu \mathrm{m}$ thin films. In other words, the effective thermal conductivity of AlN is strongly reduced in films with thickness comparable to or smaller than such long phonon MFPs, as illustrated earlier in Fig. 4(b).

We define the phonon MFP corresponding to $50 \%$ or $90 \%$ of the cumulative heat conduction as MFP (50\% or $90 \%$ ), plotting it at higher temperatures in Fig. 5(c). As the temperature increases, phonon occupation and phonon-phonon scattering increase and thus MFP (50\% or $90 \%)$ decreases. This implies that "size effects" on the thermal conductivity of AlN become somewhat less 

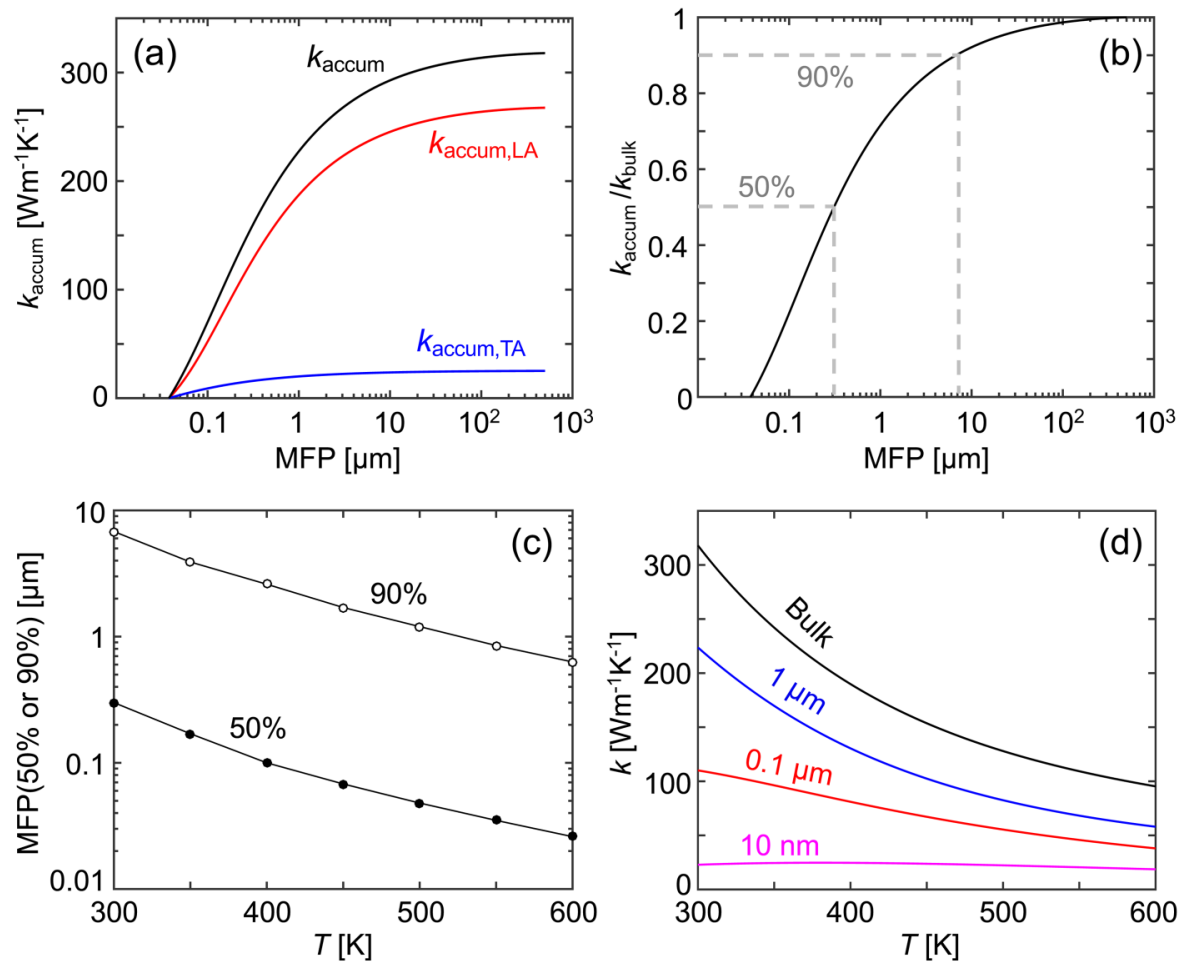

FIG. 5. (a) Calculated accumulated thermal conductivity vs phonon MFP for AIN bulk at room temperature, comparing the total and its longitudinal acoustic and transverse acoustic phonon contributions, $\quad k_{\text {accum }}=k_{\text {accum,LA }}+2 k_{\text {accum, TA }}$. (b) Normalized accumulated thermal conductivity $k_{\text {accum }} / k_{\text {bulk }}$ at room temperature, where $k_{\text {buk }}$ is the maximum value of $k_{\text {accum. }}$. Phonons with MFP larger than $0.3 \mu \mathrm{m}$ (or $7 \mu \mathrm{m}$ ) are estimated to contribute $50 \%$ (or $10 \%$ ) of the heat conduction, as shown by dashed lines. (c) Calculated temperature dependence of MFP $(50 \%$ or $90 \%)$ for AIN. (d) Expected temperature dependence of thermal conductivity for different film thicknesses, as labeled. Thinner films have weaker temperature dependence, due to the predominance of boundary scattering. All calculations [(a)-(d)] in this figure assume defect-free samples.

important at elevated temperature, i.e., the reduction of thermal conductivity in thin films of this material will be less pronounced vs the bulk value at that temperature. The thermal conductivity of thin films at high temperatures will also experience a competition between phonon-phonon and phonon-boundary scattering. This is illustrated in Fig. 5(d), which shows the expected temperature dependence of thermal conductivity from bulk to $1 \mu \mathrm{m}, 0.1 \mu \mathrm{m}$, and $10 \mathrm{~nm}$ thin films. The increasing role of phonon-boundary scattering not only lowers the thermal conductivity but also renders it less temperature-sensitive in the thinnest films and less dependent on the film thickness at the highest temperatures. The exact details of boundary scattering processes will depend, in part, on the particular surface roughness of such AlN films. These details were previously studied for $\mathrm{Si}, \mathrm{Ge}$, and $\mathrm{GaAs}$ thin films and nanowires ${ }^{62,63}$ and should be the subject of future work for AlN.

\section{CONCLUSIONS}

In summary, we have performed $3 \omega$ measurements of thermal conductivity in single crystal AlN samples from $100 \mathrm{~K}$ to $400 \mathrm{~K}$. We compared these results with analytic and $a b$ initio simulations to estimate the impurity defect densities. Aluminum vacancies play the most important role among all atomic-scale defects due to the large atomic mass mismatch, which can be analytically captured by modeling phonon-vacancy scattering using six times the mass of the missing atom. The accumulated thermal conductivity shows that phonons with MFPs larger than $0.3 \mu \mathrm{m}$ (or $7 \mu \mathrm{m}$ ) contribute to $50 \%$ (or $10 \%$ ) of heat conduction at room temperature. This implies that AlN thin films and devices with submicrometer features will exhibit strongly reduced effective thermal conductivity compared to the bulk value, even in the absence of point defects. These results are essential for understanding thermal transport in AlN thin films and devices over a broad temperature range, for applications in power electronics and deep-UV lasers.

\section{SUPPLEMENTARY MATERIAL}

See the supplementary material for additional details of the fabrication process, TDTR measurement, analytical model, and first-principles calculations.

\section{ACKNOWLEDGMENTS}

This work was supported in part by the National Science Foundation (NSF) DMREF program through Grant Nos. 1534279 and 1534303, by the NSF Engineering Research Center for Power Optimization of Electro-Thermal Systems (POETS) with Cooperative Agreement (No. EEC-1449548), and by the Stanford SystemX Alliance. This work was also supported by ASCENT, one of six centers in JUMP, a SRC program sponsored by DARPA. The experiments were performed in part at the Stanford Nanofabrication Facility and the Stanford Nano Shared Facilities, which receive funding from the NSF as part of the National Nanotechnology Coordinated Infrastructure Award (No. ECCS-1542152). A.K. and N.M. acknowledge support from the Air Force Office of Scientific Research through Grant No. FA9550615-1-0187 DEF. A.K. also acknowledges DST-INSPIRE Grant, India (Grant No. IFA17-MS122). R.L.X. and M.M.R. gratefully acknowledge technical discussions with C. Dames, V. Mishra, and W. Hodges. 


\section{REFERENCES}

'Z. Hu, K. Nomoto, W. Li, Z. Zhang, N. Tanen, Q. T. Thieu, K. Sasaki, A. Kuramata, T. Nakamura, D. Jena, and H. G. Xing, Appl. Phys. Lett. 113(12), 122103 (2018).

${ }^{2}$ J. Encomendero, R. Yan, A. Verma, S. Islam, V. Protasenko, S. Rouvimov, P. Fay, D. Jena, and H. G. Xing, Appl. Phys. Lett. 112(10), 103101 (2018).

${ }^{3}$ G. Li, B. Song, S. Ganguly, M. Zhu, R. Wang, X. Yan, J. Verma, V. Protasenko, H. G. Xing, and D. Jena, Appl. Phys. Lett. 104(19), 193506 (2014).

${ }^{4}$ S. Islam, V. Protasenko, K. Lee, S. Rouvimov, J. Verma, H. Xing, and D. Jena, Appl. Phys. Lett. 111(9), 091104 (2017).

${ }^{5}$ D. Bayerl, S. Islam, C. M. Jones, V. Protasenko, D. Jena, and E. Kioupakis, Appl. Phys. Lett. 109(24), 241102 (2016).

${ }^{6}$ E. Pop, S. Sinha, and K. E. Goodson, Proc. IEEE 94(8), 1587 (2006).

${ }^{7}$ R. Gaska, A. Osinsky, J. Yang, and M. S. Shur, IEEE Electron Device Lett. 19(3), 89 (1998)

${ }^{8}$ M. Kuball, S. Rajasingam, A. Sarua, M. Uren, T. Martin, B. Hughes, K. Hilton, and R. Balmer, Appl. Phys. Lett. 82(1), 124 (2003).

${ }^{9}$ H. Wang, M. Liserre, F. Blaabjerg, P. de Place Rimmen, J. B. Jacobsen, T. Kvisgaard, and J. Landkildehus, IEEE J. Emerging Sel. Top. Power Electron. 2(1), 97 (2014).

${ }^{10}$ G. Lang, B. Fehder, and W. Williams, IEEE Trans. Electron Devices 17(9), 787 (1970).

${ }^{11}$ M. Feneberg, R. A. Leute, B. Neuschl, K. Thonke, and M. Bickermann, Phys. Rev. B 82(7), 075208 (2010).

${ }^{12}$ J. Li, K. Nam, M. Nakarmi, J. Lin, H. Jiang, P. Carrier, and S.-H. Wei, Appl. Phys. Lett. 83(25), 5163 (2003).

${ }^{13}$ W. Yim, E. Stofko, P. Zanzucchi, J. Pankove, M. Ettenberg, and S. Gilbert, J. Appl. Phys. 44(1), 292 (1973).

${ }^{14}$ H. Okumura, S. Suihkonen, J. Lemettinen, A. Uedono, Y. Zhang, D. Piedra, and T. Palacios, Jpn. J. Appl. Phys. 57(4S), 04FR11 (2018).

${ }^{15}$ A. G. Baca, A. M. Armstrong, A. A. Allerman, E. A. Douglas, C. A. Sanchez, M. P. King, M. E. Coltrin, T. R. Fortune, and R. J. Kaplar, Appl. Phys. Lett. 109(3), 033509 (2016).

${ }^{16}$ C. Y. Ho, R. W. Powell, and P. E. Liley, J. Phys. Chem. Ref. Data 1(2), 279 (1972).

${ }^{17}$ M. Holland, Phys. Rev. 134(2A), A471 (1964).

${ }^{18}$ R. Carlson, G. Slack, and S. Silverman, J. Appl. Phys. 36(2), 505 (1965).

${ }^{19}$ G. A. Slack, J. Appl. Phys. 35(12), 3460 (1964).

${ }^{20} \mathrm{C}$. Dames, Science 361(6402), 549 (2018).

${ }^{21}$ S. Li, Q. Zheng, Y. Lv, X. Liu, X. Wang, P. Y. Huang, D. G. Cahill, and B. Lv, Science 361(6402), 579 (2018).

${ }^{22}$ H. Wada and T. Kamijoh, Jpn. J. Appl. Phys. 35(5B), L648 (1996).

${ }^{23}$ A. D. McConnell, S. Uma, and K. E. Goodson, J. Microelectromech. Syst. 10(3), 360 (2001).

${ }^{24}$ A. M. Hofmeister, Phys. Chem. Miner. 41(5), 361 (2014).

${ }^{25}$ D. G. Cahill, Rev. Sci. Instrum. 61(2), 802 (1990).

${ }^{26}$ S.-M. Lee and D. G. Cahill, J. Appl. Phys. 81(6), 2590 (1997).

${ }^{27}$ E. Sichel, R. Miller, M. Abrahams, and C. Buiocchi, Phys. Rev. B 13(10), 4607 (1976).

${ }^{28}$ G. A. Slack, R. A. Tanzilli, R. Pohl, and J. Vandersande, J. Phys. Chem. Solids 48(7), 641 (1987).

${ }^{29}$ R. Rounds, B. Sarkar, D. Alden, Q. Guo, A. Klump, C. Hartmann, T. Nagashima, R. Kirste, A. Franke, M. Bickermann, Y. Kumagai, Z. Sitar, and R. Collazo, J. Appl. Phys. 123(18), 185107 (2018).

${ }^{30}$ See http://www.hexatechinc.com/aln-growth-process.html for more information about AlN growth process (last accessed 7 December 2018).

${ }^{31}$ B. E. Gaddy, Z. Bryan, I. Bryan, J. Xie, R. Dalmau, B. Moody, Y. Kumagai, T. Nagashima, Y. Kubota, T. Kinoshita, A. Koukitu, R. Kirste, Z. Sitar, R. Collazo, and D. L. Irving, Appl. Phys. Lett. 104(20), 202106 (2014).

${ }^{32}$ C. Dames, Ann. Rev. Heat Transfer 16, 7 (2013).

${ }^{33}$ C. Dames and G. Chen, Rev. Sci. Instrum. 76(12), 124902 (2005).
${ }^{34}$ M. T. Barako, A. Sood, C. Zhang, J. Wang, T. Kodama, M. Asheghi, X. Zheng, P. V. Braun, and K. E. Goodson, Nano Lett. 16(4), 2754 (2016).

${ }^{35}$ J. Zou, D. Kotchetkov, A. Balandin, D. Florescu, and F. H. Pollak, J. Appl. Phys. 92(5), 2534 (2002).

${ }^{36}$ D. G. Cahill, Rev. Sci. Instrum. 75(12), 5119 (2004).

${ }^{37}$ A. Sood, J. Cho, K. D. Hobart, T. I. Feygelson, B. B. Pate, M. Asheghi, D. G. Cahill, and K. E. Goodson, J. Appl. Phys. 119(17), 175103 (2016).

${ }^{38}$ A. Sood, R. Cheaito, T. Bai, H. Kwon, Y. Wang, C. Li, L. Yates, T. Bougher, S. Graham, M. Asheghi, M. Goorsky, and K. E. Goodson, Nano Lett. 18, 3466 (2018).

${ }^{39}$ W. Liu and A. A. Balandin, J. Appl. Phys. 97(7), 073710 (2005).

${ }^{40}$ A. Sztein, J. Haberstroh, J. E. Bowers, S. P. DenBaars, and S. Nakamura, J. Appl. Phys. 113(18), 183707 (2013).

${ }^{41}$ D. Nilsson, E. Janzén, and A. Kakanakova-Georgieva, J. Phys. D Appl. Phys. 49(17), 175108 (2016).

${ }^{42}$ N. Katcho, J. Carrete, W. Li, and N. Mingo, Phys. Rev. B 90(9), 094117 (2014).

${ }^{43}$ A. Katre, J. Carrete, B. Dongre, G. K. Madsen, and N. Mingo, Phys. Rev. Lett. 119(7), 075902 (2017).

${ }^{44}$ A. Katre, J. Carrete, T. Wang, G. K. Madsen, and N. Mingo, Phys. Rev. Mater. 2(5), 050602 (2018).

${ }^{45}$ L. Lindsay, D. Broido, and T. Reinecke, Phys. Rev. B 87(16), 165201 (2013).

${ }^{46}$ A. Togo, F. Oba, and I. Tanaka, Phys. Rev. B 78(13), 134106 (2008).

${ }^{47}$ W. Li, J. Carrete, N. A. Katcho, and N. Mingo, Comput. Phys. Commun. 185(6), 1747 (2014).

${ }^{48}$ G. Kresse and D. Joubert, Phys. Rev. B 59(3), 1758 (1999).

${ }^{49}$ J. Carrete, B. Vermeersch, A. Katre, A. van Roekeghem, T. Wang, G. K. Madsen, and N. Mingo, Comput. Phys. Commun. 220, 351 (2017).

${ }^{\mathbf{5 0}}$ A. Katre, A. Togo, I. Tanaka, and G. K. Madsen, J. Appl. Phys. 117(4), 045102 (2015).

${ }^{\mathbf{5 1}}$ G. Slack, J. Phys. Chem. Solids 34(2), 321 (1973).

${ }^{52} \mathrm{P}$. Kuo, G. Auner, and Z. Wu, Thin Solid Films 253(1-2), 223 (1994).

${ }^{53}$ Y. Shen, J. Gaskins, X. Xie, B. M. Foley, R. Cheaito, P. E. Hopkins, and J. C. Campbell, J. Lightwave Technol. 35(19), 4242 (2017).

${ }^{54}$ C. Duquenne, M.-P. Besland, P. Tessier, E. Gautron, Y. Scudeller, and D. Averty, J. Phys. D Appl. Phys. 45(1), 015301 (2011).

${ }^{55}$ Y. Zhao, C. Zhu, S. Wang, J. Tian, D. Yang, C. Chen, H. Cheng, and P. Hing, J. Appl. Phys. 96(8), 4563 (2004).

${ }^{56}$ S. R. Choi, D. Kim, S.-H. Choa, S.-H. Lee, and J.-K. Kim, Int. J. Thermophys. 27(3), 896 (2006).

${ }^{57}$ E. Yalon, O. B. Aslan, K. K. Smithe, C. J. McClellan, S. V. Suryavanshi, F. Xiong, A. Sood, C. M. Neumann, X. Xu, K. E. Goodson, T. F. Heinz, and E. Pop, ACS Appl. Mater. Interfaces 9(49), 43013 (2017).

${ }^{58}$ A. Jacquot, B. Lenoir, A. Dauscher, P. Verardi, F. Craciun, M. Stölzer, M. Gartner, and M. Dinescu, Appl. Surf. Sci. 186(1-4), 507 (2002).

${ }^{59}$ Y. Bian, M. Liu, G. Ke, Y. Chen, J. DiBattista, E. Chan, and Y. Yang, Surf. Coat. Technol. 267, 65 (2015).

${ }^{60}$ J. T. Gaskins, P. E. Hopkins, D. R. Merrill, S. R. Bauers, E. Hadland, D. C. Johnson, D. Koh, J. H. Yum, S. Banerjee, and B. J. Nordell, ECS J. Solid State Sci. Technol. 6(10), N189 (2017).

${ }^{61}$ J. P. Freedman, J. H. Leach, E. A. Preble, Z. Sitar, R. F. Davis, and J. A. Malen, Sci. Rep. 3, 2963 (2013).

${ }^{62}$ P. N. Martin, Z. Aksamija, E. Pop, and U. Ravaioli, Nano Lett. 10(4), 1120 (2010).

${ }^{63}$ J. Carrete, L. J. Gallego, L. M. Varela, and N. Mingo, Phys. Rev. B 84(7), 075403 (2011).

${ }^{64}$ I. Kudman and E. Steigmeier, Phys. Rev. 133(6A), A1665 (1964).

${ }^{65}$ A. Jeżowski, P. Stachowiak, T. Plackowski, T. Suski, S. Krukowski, M. Boćkowski, I. Grzegory, B. Danilchenko, and T. Paszkiewicz, Phys. Status Solidi 240(2), 447 (2003).

${ }^{66}$ C. Mion, J. Muth, E. Preble, and D. Hanser, Appl. Phys. Lett. 89(9), 092123 (2006).

${ }^{67}$ Z. Guo, A. Verma, X. Wu, F. Sun, A. Hickman, T. Masui, A. Kuramata, M. Higashiwaki, D. Jena, and T. Luo, Appl. Phys. Lett. 106(11), 111909 (2015). 